\title{
GENERALIZATION OF SUM REPRESENTATION FUNCTIONAL EQUATIONS-I
}

\author{
PL. KANNAPPAN \\ Faculty of Mathematics \\ University of Waterloo \\ Waterloo, Ontario, Canada \\ and \\ V. SATHYABHAMA \\ Department of Mathematics \\ University of Kerala \\ Trivandrum, Kerala, India
}

(Received September 20, 1977 and in revised form March 3, 1978)

ABSTRACT. This paper contains the solution of a functional equation which is a generalization of a functional equation arising in a characterezation of directed divergence and inaccuracy.

KEY WORDS AND PHRASES. Functional Equations, Shannon's Entropy, Probability vistributions, and Characterization of Directed Divergence and Inaccuracy.

AMS (MOS) SUBJECT CLASSIFICATION (1970) CODES. 39A15, 94A15

1. INTRODUCTION.

It was shown in [7] that the properties of symmetry, expansibility and branching (possessed by directed divergence and inaccuracy) lead to a sum representation of the measures which together with the property of additivity leads to the study of the functiona1 equation 


$$
\sum_{i=1}^{n} \sum_{j=1}^{m} f\left(x_{1} y_{j}, u_{i} v_{j}\right)=\sum_{i=1}^{n} f\left(x_{1}, u_{i}\right)+\sum_{j=1}^{m} f\left(y_{j}, v_{j}\right)
$$

For more information on (1.1) refer to $[2,3,4]$. Here we consider the following functional equation,

$$
\sum_{i=1}^{2} \sum_{j=1}^{3} F_{i j}\left(p_{i} r_{j}, q_{1} s_{j}\right)=\sum_{i=1}^{2} G_{i}\left(p_{i}, q_{i}\right)+\sum_{j=1}^{3} H_{j}\left(r_{j}, s_{j}\right),
$$

which is a generalization of the equation (1.1) (for $n=2, m=3$ ) and solve (1.2) by employing a technique similar to that in [6] used to solve a generalized form similar to (1:1) connected with Shannon's entrophy.

\section{MAIN RESULTS.}

Let $\Delta_{n}=\left\{P=\left(p_{1}, \ldots P_{n}\right): P_{n} \geq 0, \sum_{i=1}^{n} P_{1}=1\right\}$ denote the set of all complete finite n-ary probability distributions. Let $P, Q, \epsilon \Delta_{2}, R, S \in \Delta_{3}$. Let $J=] 0,1[x] 0,1\left[\cup\{(0, y)\} \cup\{(1, z)\}\right.$ with $y \in\left[0,1[\right.$ and $z \in 10,1]$. Let $F_{1 j}, G_{1}, H_{j}$ : $J \rightarrow R$ (reals) be measurable in each variable and satisfy the function equation (1.2), We obtain the solution of the functional equation (1.2) through a sequence of auxiliary results, which we state in the following propositions.

REMARK. Here we are solving (1.2) for complete probability distributions, which differs from the previous works [see 2,3 etc.] based on incomplete distributions $\left(\Sigma u_{1} \leq 1, \Sigma v_{j} \leq 1\right)$. This fills the existing gap satisfactorily.

PROPOSITION 1. Let $F: J \rightarrow R$ be measurable in each variable and satisfy the functional equation

$$
F(p, q)-F(p x, q y)-F((1-x) p,(1-y) q)=A(p, q, x, y),
$$

where

$$
A\left(p_{1}+p_{2}, q_{1}+q_{2}, x, y\right)=A\left(p_{1}, q_{1}, x, y\right)+A\left(p_{2}, q_{2}, x, y\right)
$$

for every fixed $x, y \in[0,1]$ and

$$
F(1,1)=0 \text {. }
$$


Then the solutions of equation (2.1) are given by:

$$
\begin{aligned}
\mathrm{F}(\mathrm{p}, \mathrm{q}) & =a p \log \mathrm{p}+\mathrm{bp} \log \mathrm{q}+\mathrm{cq} \log q+\mathrm{a}^{\prime} \mathrm{p}-\mathrm{a}^{\prime} \mathrm{q} \\
\mathrm{A}(\mathrm{p}, \mathrm{q}, \mathrm{x}, \mathrm{y}) & =\mathrm{p}[\mathrm{aI}(\mathrm{x}, \mathrm{x})+\mathrm{bI}(\mathrm{x}, \mathrm{y}]+\mathrm{cqI}(\mathrm{y}, \mathrm{y})
\end{aligned}
$$

where

$$
I(x, y):=-x \log y-(1-x) \log (1-y)
$$

This result follows easily from [5], by verifying that $F$ in (2.1) satisfies (1.1) for $m=2, n=3$, by using $(2.2)$ and the condition $F(1,1)=0$.

PROPOSITION 2. Let $F: J \rightarrow R$ be measurable in each variable and satisfy the functional equation (2.1), where $A(p, q, x, y)$ satisfies (2.2). Then, the solutions of equation (2.1) are given by

$$
\begin{aligned}
F(p, q) & =a p \log p+b p \log q+c q \log q+c_{1} p+c_{2} q \\
A(p, q, x, y) & =p[a I(x, x)+b I(x, y)]+c q I(y, y) .
\end{aligned}
$$

PROOF. Define $G(p, q)=F(p, q)+d p+e q$, where $d+e=-F(1,1)$.

Since $F$ is measurable in each variable, $G$ is measurable in each variable. Also, $G(p, q)$ satisfies the functional equation $(2.1)$ and $G(1,1)=0$. Hence, by proposition 1, we have,

$$
G(p, q)=a p \log p+b p \log q+c q \log q+a^{\prime} p-a^{\prime} q^{\prime \prime}
$$

so that,

$$
F(p, q)=a p \log p+b p \log q+c q \log q+c_{1} p+c_{2} q
$$

and

$$
A(p, q, x, y)=p[a I(x, x)+b I(x, y)]+c q I(y, y)
$$

PROPOSITION 3. Let the function $F: J \rightarrow R$ be measurable in each variable and satisfy the equation

$$
F(p, q)-F(p x, q y)-F((1-x) p,(1-y) q)=A(p, q, x, y)+B(x, y) \text {, }
$$


where $(p, q) \rightarrow A(p, q, x, y)$ satisfies (2.2). Then the solutions of equation (2.3) are given by

$$
\begin{gathered}
B(x, y)=c_{3}, \\
F(p, q)=a p \log p+b p \log q+c q \log q+c_{1} p+c_{2} q-c_{3}, \\
A(p, q, x, y)=p[a I(x, x)+b I(x, y)]+c q I(y, y),
\end{gathered}
$$

where $c_{3}=-F(0,0)$.

PROOF. Putting $p=0=q$ in equation (2.3), we get

$$
-F(0,0)=B(x, y) \quad,
$$

that is,

$$
B(x, y)=c_{3} \text {, say. }
$$

We now define $G(p, q)=F(p, q)+c_{3}$. Then $G(p, q)$ satisfies the equation (2.1). Also, since $F$ is measurable in both the variables, $G$ is measurable in both variables. Hence, by proposition 2, we get

$$
G(p, q)=a p \log p+b p \log q+c q \log q+c_{1} p+c_{2} q
$$

so that

$$
F(p, q)=a p 108 p+b p \log q+c q 108 q+c_{1} p+c_{2} q-c_{3}
$$

and

$$
A(p, q, x, y)=p[a I(x, x)+b I(x, y)]+c q I(y, y)
$$

and the proof of prope ition 3 is complete.

PROPOSITION 4. Let $F_{1}: J \rightarrow R,(i=1,2,3)$ be measurable in each variable satisfying the functional equation

$F_{1}(p, q)-F_{2}(p x, q y)-F_{3}((1-x) p,(1-y) q)=A(p, q, x, y)+B(x, y)$,

where $(P, q) \quad A(p, q, x, y)$ satisfies (2.2). Then the solutions of equation (2.4) 
are given by

$$
\begin{aligned}
& F_{1}(p, q)=a p \log p+b p \log q+c q \log q+c_{1} p+c_{2} q+d_{1} \\
& F_{2}(p, q)=a p \log p+b p \log q+c q \log q+\left(c_{1}-c_{3}\right) p+\left(c_{2}-c_{4}\right) q+d_{2}, \\
& F_{3}(p, q)=a p \log p+b p \log q+c q \log q+\left(c_{1}-c_{5}\right) p+\left(c_{2}-c_{6}\right) q+d_{3}, \\
& \begin{aligned}
A(p, q, x, y)=p[a I(x, x)+b I(x, y)]+c q I(y, y)+\left(c_{3}-c_{5}\right) p x+ \\
+\left(c_{4}-c_{6}\right) q y+c_{5} p+c_{6} q
\end{aligned} \\
& B(x, y)=d_{1}-d_{2}-d_{3} .
\end{aligned}
$$

PROOF. Putting $p=0=q$ in the equation (2.4), we get

$$
F_{1}(0,0)-F_{2}(0,0)-F_{3}(0,0)=B(x, y) .
$$

Denoting $F_{i}(0,0)=d_{i}(i=1,2,3),(2.6)$ yields,

$$
d_{1}-d_{2}-d_{3}=B(x, y)
$$

Equation (2.4) with $x=1=y$, by means of (2.7), gives

$$
F_{1}(p, q)-F_{2}(p, q)-F_{3}(0,0)=A(p, q, 1,1)+d_{1}-d_{2}-d_{3} \text {. }
$$

That is,

$$
F_{2}(p, q)=F_{1}(p, q)-A(p, q, 1,1)+d_{2}-d_{1} \text {. }
$$

When $x=0=y$, we get frez equation (2.4),

$$
F_{3}(p, q)=F_{1}(p, q)-A(p, q, 0,0)+d_{3}-d_{1} \text {. }
$$

Substituting for $F_{2}$ and $F_{3}$ from (2.8) and (2.9) into (2.4), we obtain,

$$
\begin{array}{r}
F_{1}(p, q)-F_{1}(p x, q y)-F_{1}((1-x) p,(1-y) q)=A(p, q, x, y)-A(p x, q y, 1,1)-A((1-x) p, \\
(1-y) q, 0,0)-d_{1}=A_{1}(p, q, x, y)-d_{1},
\end{array}
$$

where

$$
A_{1}(p, q, x, y)=A(p, q, x, y)-A(p x ; q y, 1,1)-A((1-x) p,(1-y) q, 0,0),
$$

and $(p, q) \rightarrow A_{1}(p, q, x, y)$ satisfies (2.2). 
Hence, applying proposition 3 to equation (2.10), we get

$$
\begin{aligned}
& F_{1}(p, q)=a p \log p+b p \log q+c q \log q+c_{1} p+c_{2} q+d_{1}, \\
& A_{1}(p, q, x, y)=p[a I(x, x)+b I(x, y)]+c q I(y, y),
\end{aligned}
$$

from which using (2.11) we get,

$A(p, q, x, y)=p[a I(x, x)+b I(x, y)]+A(p x, q y, 1,1)+A((1-x) p,(1-y) q, 0,0)$.

Since $F_{1}(p, q)(i=1,2,3)$ is measurable in each variable, $A(p, q, 1,1)$ is measurable in the first and the second co-ordinates. Since $(p, q) \rightarrow A(p, q, 1,1)$ satisfies the relation $(2.2)$, we have

$A(p, q, 1,1)+A(r, s, 1,1)+A(p+r, q+s, 1,1),(p, q),(r, s) \in J, p, q, r, s \in[0,1]$. (2.14)

When $p=0=r$, in the equation (2.14), we get

$$
A(0, q, 1,1)+A(0, s, 1,1)=A(0, q+8,1,1), q, s, q+8 \in[0,1[.
$$

Since $A(0, \cdot, 1,1)$ is measurable, $[1, p p .8-9]$, we have, $A(0, q, 1,1)=c_{4} q$, where $c_{4}$ is an arbitrary constant. If we define $B(p, q)=A(p, q, 1,1)-c_{4} q$, then $B(0, q)=0$ and equation (2.14) becomes

$$
\beta(p, q)+\beta(r, s)=\beta(p+r, q+s),(p, q),(r, s) \in J, p, q, r, s \in[0,1] .
$$

Putting $p=0$ in $(2.16)$, we get

$$
\beta(r, s)=\beta(r, q+s),
$$

that 1s, $\beta(r, s)=$ constant, Independent of $s$, say $\theta(r)$. Thus, from (2.16) we obtain,

$$
\theta(p)+\theta(r)=\theta(p+r), \quad p, r \in[0,1]
$$

But $A(p, q, 1,1)$ is measurable in the first and second co-ordinates, and hence $\beta(p, q)$ is measurable in $p$ and $q$, from which it follows that $\theta(p)$ is measurable. Thus, from $[1]$ again we have, $\theta(p)=c_{3} p$, where $c_{3}$ is an arbitrary constant. That 1s, $\theta(p)=\beta(p, q)=c_{3} p=A(p, q, 1,1)-c_{4} q$, from which it follows that 


$$
A(p, q, 1,1)=c_{3} p+c_{4} q,
$$

where $c_{3}$ snd $c_{4}$ are arbitrary constants. Similarly, we can show that

$$
A(p, q, 0,0)=c_{5} p+c_{6} q
$$

Where $c_{5}$ and $c_{6}$ are arbirtaty constants. From $(2.13),(2.19)$ and $(2.20)$ we get,

$$
\begin{aligned}
A(p, q, x, y) & =p[a I(x, x)+b I(x, y)]+c_{3} p x+c_{4} q y+c_{5}(1-x) p+c_{6}(1-y) q+c q I(y, y), \\
& =p[a I(x, x)+b I(x, y)]+\left(c_{3}-c_{5}\right) p x+\left(c_{4}-c_{6}\right) q y+c_{5} p+c_{6} q+c q I(y, y) .
\end{aligned}
$$

From (2.8), (2.12) and (2.19) we obtain,

$$
F_{2}(p, q)=a p \log p+b p \log q+c q \log q+\left(c_{1}-c_{3}\right) p+\left(c_{2}-c_{4}\right) q+d_{2} \text {, }
$$

and from (2.9), (2.12) and (2.20) we get,

$$
F_{3}(p, q)=a p \log p+b p \log q+c q \log q+\left(c_{1}-c_{5}\right) p+\left(c_{2}-c_{6}\right) q+d_{3} \text {. }
$$

Now, $(2.12),(2.22),(2.23),(2.21)$ and (2.7) yield the solutions (2.5) and the proof of proposition 4 is complete.

PROPOSITION 5. Let the functions $F_{1}: J \rightarrow R(1=1,2, \ldots, 6)$ which are measurable in each variable satisfy the functional equation

$$
\begin{aligned}
& {\left[F_{1}\left(p_{1}, q_{1}\right)-F_{2}\left(p_{1} x, q_{1} y\right)-F_{3}\left((1-x) p_{1},(1-y) q_{1}\right)\right]} \\
& +\left[F_{4}\left(p_{2}, q_{2}\right)-F_{5}\left(p_{2} x, q_{2} y\right)-F_{6}\left((1-x) p_{2},(1-y) q_{2}\right)\right] \\
& =A\left(p_{1}+p_{2}, q_{1}+q_{2}, x, y\right), p_{1}+p_{2} \in[0,1], q_{1}+q_{2} \in[0,1]
\end{aligned}
$$

Then the solutions of the equation $(2.24)$ are given by

$$
\begin{aligned}
& F_{1}(p, q)=a p \log p+b p \log q+c q \log q+c_{1} p+c_{2} q+d_{1}, \\
& F_{2}(p, q)=a p \log p+b p \log q+c q \log q+\left(c_{1}-c_{3}\right) p+\left(c_{2}-c_{4}\right) q+d_{2}, \\
& F_{3}(p, q)=a p \log p+b p \log q+c q \log q+\left(c_{1}-c_{5}\right) p+\left(c_{2}-c_{6}\right) q+d_{3}, \\
& F_{4}(p, q)=a p \log p+b p \log q+c q \log q+c_{1}^{\prime} p+c_{2}^{\prime} q+d_{4}, \\
& F_{5}(p, q)=a p \log p+b p \log q+c q \log q+\left(c_{1}^{\prime}-c_{3}\right) p+\left(c_{2}^{\prime}-c_{4}\right) q+d_{5}, \\
& F_{6}(p, q)=a p \log p+b p \log q+c q \log q+\left(c_{1}^{\prime}-c_{5}\right) p+\left(c_{2}^{\prime}-c_{6}\right) q+d_{6},
\end{aligned}
$$




$$
\begin{aligned}
A(p, q, x, y)= & p(a I(x, x)+b I(x, y))+c q I(y, y)+\left(c_{3}-c_{5}\right) d x+\left(c_{4}-c_{6}\right) q y \\
& +c_{5} p+c_{6} q+d_{1}-d_{2}-d_{3}+d_{4}-d_{5}-d_{6} .
\end{aligned}
$$

PROOF. Putting $\mathrm{p}_{2}=\mathrm{q}_{2}=0$ in the equation (2.24), we get,

$$
\begin{aligned}
F_{1}\left(p_{1}, q_{1}\right) & -F_{2}\left(p_{1} x, q_{1} y\right)-F_{3}\left((1-x) p_{1},(1-y) q_{1}\right)+F_{4}(0,0) \\
& -F_{5}(0,0)-F_{6}(0,0)=A\left(p_{1}, q_{1}, x, y\right) .
\end{aligned}
$$

When $\mathrm{p}_{1}=\mathrm{q}_{1}=0$ in $(2.24)$, we have

$$
\begin{gathered}
F_{4}\left(p_{2}, q_{2}\right)-F_{5}\left(p_{2} x, q_{2} y\right)-F_{6}\left((1-x) p_{2},(1-y) q_{2}\right)+F_{1}(0,0) \\
-F_{2}(0,0)-F_{3}(0,0)=A\left(p_{2}, q_{2}, x, y\right) .
\end{gathered}
$$

Adding (2.26) and (2.27) and comparing with the equation (2.24), we get

$$
\begin{gathered}
A\left(p_{1}+p_{2}, q_{1}+q_{2}, x, y\right)+F_{1}(0,0)-F_{2}(0,0)-F_{3}(0,0)+F_{4}(0,0) \\
-F_{5}(0,0)-F_{6}(0,0)=A\left(p_{1}, q_{1}, x, y\right)+A\left(p_{2}, q_{2}, x, y\right) .
\end{gathered}
$$

Denoting $F_{1}(0,0)=d_{1} \quad(i=1,2, \ldots, 6)$ and defining

$$
A_{1}(p, q, x, y)=A(p, q, x, y)-d_{1}+d_{2}+d_{3}-d_{4}+d_{5}+d_{6} \text {, }
$$

(2.28) becomes,

$$
A_{1}\left(p_{1}+p_{2}, q_{1}+q_{2}, x, y\right)=A_{1}\left(p_{1}, q_{1}, x, y\right)+A_{1}\left(p_{2}, q_{2}, x, y\right),
$$

that is,

$$
(p, q) \rightarrow A_{1}(p, q, x, y)
$$

satisfies (2.2). Now from equation (2.26) and (2.27) we get using (2.29),

$$
\begin{gathered}
F_{1}\left(p_{1}, q_{1}\right)-F_{2}\left(p_{1} x, q_{1} y\right)-F_{3}\left((1-x) p_{1},(1-y) q_{1}\right) \\
=A_{1}\left(p_{1}, q_{1}, x, y\right)+d_{1}-d_{2}-d_{3},
\end{gathered}
$$

and

$$
F_{4}\left(p_{2}, q_{2}\right)-F_{5}\left(p_{2} x, q_{2} y\right)-F_{6}\left((1-x) p_{2},(1-y) q_{2}\right)=A_{1}\left(p_{2}, q_{2}, x, y\right)+d_{4}-d_{5}-d_{6},
$$


where $(p, q) \rightarrow A_{1}(p, q, x, y)$ satisfies (2.2). Hence, applying proposition 4 seperately to the equations $(2.30)$ and (2.31), we get,

$$
\left.\begin{array}{rl}
F_{1}(p, q) & =a p \log p+b p \log q+c q \log q+c_{1} p+c_{2} q+d_{1} \\
F_{2}(p, q) & =a p \log p+b p \log q+\left(c_{1}-c_{3}\right) p+\left(c_{2}-c_{4}\right) q+d_{2}+c q \log q \\
F_{3}(p, q) & =a p \log p+b p \log q+\left(c_{1}-c_{5}\right) p+\left(c_{2}-c_{6}\right) q+d_{3}+c q \log q, \\
A(p, q, x, y) & =p[a I(x, x)+b I(x, y)]+\left(c_{3}-c_{5}\right) p x+\left(c_{4}-c_{6}\right) q y+c q I(y, y) \\
& +c_{5} p+c_{6} q+d_{1}-d_{2}-d_{3}+d_{4}-d_{5}-d_{6},
\end{array}\right\}
$$

and

$$
\left.\begin{array}{rl}
F_{4}(p, q) & =a^{\prime} p \log p+b^{\prime} p \log q+c^{\prime} q \log q+c_{1}^{\prime} p+c_{2}^{\prime} q+d_{4}, \\
F_{5}(p, q) & =a^{\prime} p \log p+b^{\prime} p \log q+\left(c_{1}^{\prime}-c_{3}^{\prime}\right) p+\left(c_{2}^{\prime}-c_{4}^{\prime}\right) q+d_{5}+c^{\prime} q \log q \\
F_{6}(p, q) & =a^{\prime} p \log p+b^{\prime} p \log q+\left(c_{1}^{\prime}-c_{5}^{\prime}\right) p x+\left(c_{2}^{\prime}-c_{6}^{\prime}\right) q^{\prime}+d_{6}+c^{\prime} q \log q,\left(c_{3}^{\prime}-c_{5}^{\prime}\right) p x+\left(c_{4}^{\prime}-c_{6}^{\prime}\right) q y+c^{\prime} q I(y, y) \\
A(p, q, x, y) & =p\left[a^{\prime} I(x, x)+b^{\prime} I(x, y)\right]+\left(c^{\prime} y\right. \\
& +c_{5}^{\prime} p+c_{6}^{\prime} q+d_{1}-d_{2}-d_{3}+d_{4}-d_{5}-d_{6} .
\end{array}\right\}
$$

Comparing the values of $A$ in (2.32) and (2.33) we see that,

$$
a^{\prime}=a, b^{\prime}=b, c_{3}^{\prime}-c_{5}^{\prime}=c_{3}-c_{5}, c_{4}^{\prime}-c_{6}^{\prime}=c_{4}-c_{6}, c_{5}^{\prime}=c_{5}, c_{6}^{\prime}=c_{6}, c^{\prime}=c .
$$

Thus, we have $c_{3}^{\prime}=c_{3}$ and $c_{4}^{\prime}=c_{4}$. Hence, from the expressions (2.32) and (2.33), (2.25) follows and the proposition 5 is proved. The converse is also true.

Propositions 1 to 5 lead us to the following main theorem.

THEOREM. Let $F_{i j}, G_{i}, H_{j}: J \rightarrow R \quad(i=1,2 ; j=1,2,3)$ be functions which are measurable in each variable satisfying the functional equation (1.2). Then the solutions of the equation (1.2) are given by

$$
\left.\begin{array}{l}
\mathrm{H}_{1}(p, q)=a p \log p+b p \log q+c q \log q+c_{1} p+c_{2} q+e_{1}, \\
H_{2}(p, q)=a p \log p+b p \log q+c q \log q+c_{1}^{\prime} p+c_{2}^{\prime} q+e_{4}, \\
H_{3}(p, q)=a p \log p+b p \log q+c q \log q+c_{1}^{\prime \prime} p+c_{2}^{\prime \prime} q+e_{7}, \\
F_{11}(p, q)=a p \log p+b p \log q+c q \log q+\left(c_{1}-c_{3}\right) p+\left(c_{2}-c_{4}\right) q+e_{2},
\end{array}\right\}
$$




$$
\begin{aligned}
F_{12}(p, q) & =a p \log p+b p \log q+c q \log q+\left(c_{1}^{\prime}-c_{3}\right) p+\left(c_{2}^{\prime}-c_{4}\right) q+e_{5}, \\
F_{13}(p, q) & =a p \log p+b p \log q+c q \log q+\left(c_{1}^{\prime \prime}-c_{3}\right) p+\left(c_{2}^{\prime \prime}-c_{4}\right) q+e_{8}, \\
F_{21}(p, q) & =a p \log p+b p \log q+c q \log q+\left(c_{1}-c_{5}\right) p+\left(c_{2}-c_{6}\right) q+e_{3}, \\
F_{22}(p, q) & =a p \log p+b p \log q+c q \log q+\left(c_{1}^{\prime}-c_{5}\right) p+\left(c_{2}^{\prime}-c_{6}\right) q+e_{6}, \\
F_{23}(p, q)= & a p \log p+b p \log q+c q \log q+\left(c_{1}^{\prime \prime}-c_{5}\right) p+\left(c_{2}^{\prime \prime}-c_{6}\right) q+e_{9}, \\
G_{1}(p, q)= & g(p, q), \\
G_{2}(p, q)= & e_{9}+e_{8}-e_{7}-g(1-p, 1-q)-a I(p, p)-b I(p, q)-c I(q, q) \\
-\left(c_{3}-c_{5}\right)(1-p)-\left(c_{4}-c_{6}\right)(1-q) & -e_{1}+e_{2}+e_{3}-e_{4}+e_{5}+e_{6}-c_{5}-c_{6},
\end{aligned}
$$

where $g(p, q)$ is an arbitrary measureable function and $e_{i}(i=1,2, \ldots, 9), a, b, c, c_{1}$ $(i=1,2,3,4), c_{1}^{\prime}, c_{1}^{\prime \prime}, c_{2}^{\prime}$ and $c_{2}^{\prime \prime}$ are arbitrary constants.

PROOF. In the equation (1.2) put $\mathrm{p}_{1}=\mathrm{p}, \mathrm{p}_{2}=1-\mathrm{p} ; \mathrm{q}_{1}=\mathrm{q}, \mathrm{q}_{2}=1-\mathrm{q}$; $r_{3}=1-r_{2}-r_{1}, s_{3}=1-s_{1}-s_{2}$. Then we obtain,

$$
\begin{aligned}
& {\left[\mathrm{H}_{1}\left(\mathrm{r}_{1}, \mathrm{~s}_{1}\right)-\mathrm{F}_{11}\left(\mathrm{pr}_{1}, \mathrm{qs} \mathrm{s}_{1}\right)-\mathrm{F}_{21}\left((1-\mathrm{p}) \mathrm{r}_{1},(1-\mathrm{q}) \mathrm{s}_{1}\right)\right]} \\
& +\left[\mathrm{H}_{2}\left(\mathrm{r}_{2}, \mathrm{~s}_{2}\right)-\mathrm{F}_{12}\left(\mathrm{pr}_{2}, q \mathrm{~s}_{2}\right)-\mathrm{F}_{22}\left((1-\mathrm{p}) \mathrm{r}_{2},(1-\mathrm{q}) \mathrm{s}_{2}\right)\right] \\
& =\left[\mathrm{F}_{13}\left(\mathrm{pr}_{3}, \mathrm{qs} \mathrm{s}_{3}\right)+\mathrm{F}_{23}\left((1-\mathrm{p}) \mathrm{r}_{3},(1-\mathrm{q}) \mathrm{s}_{3}\right)-\mathrm{H}_{3}\left(\mathrm{r}_{3}, \mathrm{~s}_{3}\right)\right] \\
& -G_{1}(p, q)-G_{2}(1-p, 1-q) \\
& =: A\left(1-r_{3}, 1-s_{3}, p, q\right) \text {, say. }
\end{aligned}
$$

From the identity,

$$
\begin{aligned}
{\left[\mathrm{H}_{1}\left(\mathrm{r}_{1}, \mathrm{~s}_{1}\right)-\mathrm{F}_{11}\left(\mathrm{pr}_{1}, \mathrm{qs} \mathrm{s}_{1}\right)-\mathrm{F}_{21}\left((1-\mathrm{p}) \mathrm{r}_{1},(1-\mathrm{q}) \mathrm{s}_{1}\right)\right] } \\
+\left[\mathrm{H}_{2}\left(\mathrm{r}_{2}, \mathrm{~s}_{2}\right)-\mathrm{F}_{12}\left(\mathrm{pr}_{2}, \mathrm{qs_{2 }}\right)-\mathrm{F}_{22}\left((1-\mathrm{p}) \mathrm{r}_{2},(1-\mathrm{q}) \mathrm{s}_{2}\right)\right] \\
=\mathrm{A}\left(\mathrm{r}_{1}+\mathrm{r}_{2}, \mathrm{~s}_{1}+\mathrm{s}_{2}, \mathrm{p}, \mathrm{q}\right),
\end{aligned}
$$

we deduce from proposition 5 that,

$$
\left.\begin{array}{rl}
\mathrm{H}_{1}(r, s) & =\text { ar } \log r+b r \log s+c s \log s+c_{1} r+c_{2} s+e_{1}, \\
F_{11}(r, s) & =\text { ar } \log r+b r \log s+c s \log s+\left(c_{1}-c_{3}\right) r+\left(c_{2}-c_{4}\right) s+e_{2},
\end{array}\right\}
$$




$$
\begin{aligned}
\mathrm{F}_{21}(r, s)= & \text { ar } \log r+b r \log s+c s \log s+\left(c_{1}-c_{5}\right) r+\left(c_{2}-c_{6}\right) s+e_{3}, \\
H_{2}(r, s)= & \text { ar } \log r+b r \log s+c s \log s+c_{1}^{\prime} r+c_{2}^{\prime} s+e_{4}, \\
F_{12}(r, s)= & \text { ar } \log r+b r \log s+c s \log s+\left(c_{1}^{\prime}-c_{3}\right) r+\left(c_{2}^{\prime}-c_{4}\right) s+e_{5}, \\
F_{22}(r, s)= & \text { ar } \log r+b r \log s+c s \log s+\left(c_{1}^{\prime}-c_{5}\right) r+\left(c_{2}^{\prime}-c_{6}\right) s+e_{6}, \\
A(r, s, p, q)= & r[a I(p, p)+b I(p, q)]+\left(c_{3}-c_{5}\right) r p+\left(c_{4}-c_{6}\right) s q+c s I(q, q) \\
& +c_{5} r+c_{6} s+e_{1}-e_{2}-e_{3}+e_{4}-e_{5}-e_{6} .
\end{aligned}
$$

From (2.35) we have, (using (2.36)),

$$
\begin{aligned}
& \mathrm{H}_{3}\left(r_{3}, s_{3}\right)-F_{13}\left(p_{3}, q s_{3}\right)-F_{23}\left((1-p) r_{3},(1-q) s_{3}\right) \\
&=-A\left(1-r_{3}, 1-s_{3}, p, q\right)-G_{1}(p, q)-G_{2}(1-p, 1-q), \\
&=-\left(1-r_{3}\right)[a I(p, p)+b I(p, q)]-c\left(1-s_{3}\right)(q, q)-\left(c_{3}-c_{5}\right)\left(1-r_{3}\right) p \\
&-\left(c_{4}-c_{6}\right)\left(1-s_{3}\right) q-c_{5}\left(1-r_{3}\right)-c_{6}\left(1-s_{3}\right) \\
&-e_{1}+e_{2}+e_{3}-e_{4}+e_{5}+e_{6}-G_{1}(p, q)-G_{2}(1-p, 1-q) . \\
&=\left\{r_{3}[a I(p, p)+b I(p, q)]+p r_{3}\left(c_{3}-c_{5}\right)+\left(c_{4}-c_{6}\right) s_{3} q+c_{3}(q, q)\right. \\
&\left.+c_{5} r_{3}+c_{6} s_{3}\right\}-\left\{a I(p, p)+b I(p, q)+c I(q, q)+\left(c_{3}-c_{5}\right) p\right. \\
&+\left(c_{4}-c_{6}\right) q+c_{5}+c_{6}+e_{1}-e_{2}-e_{3}+e_{4}-e_{5}-e_{6} \\
&\left.+G_{1}(p, q)+G_{2}(1-p, 1-q)\right\} .
\end{aligned}
$$

Applying proposition 4 to (2.37), we get

$$
\begin{aligned}
& \mathrm{H}_{3}(r, s)=\text { ar } \log r+b r \log s+c s \log s+c_{1}^{\prime \prime} r+c_{2}^{\prime \prime} s+e_{7}, \\
& F_{13}(r, s)=\text { ar } \log r+b r \log s+c s \log s+\left(c_{1}^{\prime \prime}-c_{3}\right) r+\left(c_{2}^{\prime \prime}-c_{4}\right) s+e_{8}, \\
& F_{23}(r, s)=\text { ar } \log r+b r \log s+c s \log s+\left(c_{1}^{\prime \prime}-c_{5}\right) r+\left(c_{2}^{\prime \prime}-c_{6}\right) s+e_{9}, \\
& \text { aI }(p, p)+b I(p, q)+c I(q, q)+\left(c_{3}-c_{5}\right) p+\left(c_{4}-c_{6}\right) q+c_{5}+c_{6}+e_{1}-e_{2} \\
& -e_{3}+e_{4}-e_{5}-e_{6}+G_{1}(p, q)+G_{2}(1-p, 1-q)=e_{9}+e_{8}-e_{7} .
\end{aligned}
$$

Let $G_{1}(p, q)=g(p, q)$. Then $g$ is measurable in each variable. Thus, from the last expression in (2.38), we get 


$$
\begin{aligned}
G_{2}(p, q)= & e_{9}+e_{8}-e_{7}-g(1-p, 1-q)-a I(p, p)-b I(p, q)-c I(q, \grave{q}) \\
- & \left(c_{3}-c_{5}\right)(1-p)-\left(c_{4}-c_{6}\right)(1-q)-e_{1}+e_{2}+e_{3} \\
& -e_{4}+e_{5}+e_{6}-c_{5}-c_{6} .
\end{aligned}
$$

Now (2.36), (2.38) and (2.39) give the solutions (2.34). This completes the proof of the theorem.

One can easily verify that the eleven functions given by the expressions (2.34) satisfy the functional equation (1.2).

\section{APPLICATION.}

As pointed out in section one, from the solutions of the functional equation (1.2) and the sum representation of the measures and some initial conditions ${ }^{-}[2]$, we can obtain the measures the directed divergence and the inaccuracy.

ADDITION: REMARK. In almost every characterization of the measures, the Shannon entropy, the directed divergence, the inaccuracy etc., several functional equations are used to acheive the result. In one set of characterizations, one comes across equations of the type

$$
\text { (*) } f(x)+(1-x) f\left(\frac{y}{1-x}\right)=f(y)+(1-y) f\left(\frac{x}{1-y}\right)
$$

and in the other, one comes across the functional equations of the type (1.1). Recently, in dealing with the mixed theory of information (as distinguished from the probabilistic and nonprobabilistic theories), we came across generalization of (*) of the form

$$
f(x)+(1-x) g\left(\frac{y}{1-x}\right)=h(y)+(1-y) k\left(\frac{x}{1-y}\right) .
$$

In a similar manner, while considering sum representation forms in the mixed theory of information, one might have to deal with functional equations of the form (1.2), which is a generalization of the form (1.1) 
ACKNOWLEDGMENT. This research is partially supported by a NSERC of Canada grant.

\section{REFERENCES}

1. Aczel, J., and Z. Daroczy, On measures of information and their characterizations, Academic Press, (1975).

2. Kannappan, PL., On Shannon's entropy, directed divergence and inaccuracy, Z. Wahr. Verw. Geb. 22 (1972) 95-100.

3. Kannappan, PL., On directed divergence and inaccuracy, Z. Wahr. Verw. Geb. $\underline{25}$ (1972) 49-55.

4. Kannappan, PL., On the Measurable Solutions of two functional equations, Mathamatica Cluj., 16 (39) (1974), no 2 (1977), 275-280.

5. Kannappan, PL., and P. N. Rathie, On the measureable solution of a functional equation in two variables (to appear).

6. $\mathrm{Ng}, \mathrm{C} . \mathrm{T}$. , On the Measurable solutions of the functional equation

233

$\sum_{i=1} \sum_{j=1} F_{i}, j\left(p, q_{j}\right)=\sum_{i=1} G_{i}\left(p_{i}\right)+\sum_{j=1} H_{j}\left(q_{j}\right)$, Acta Math. Acad. Sci. Hungar.

$\underline{25}$ (1974) 249-254.

7. Ng. C. T., Representation for measures of information with the branching property, Information and Control 25 (1974) 45-56. 


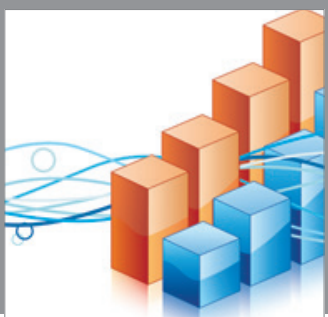

Advances in

Operations Research

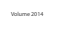

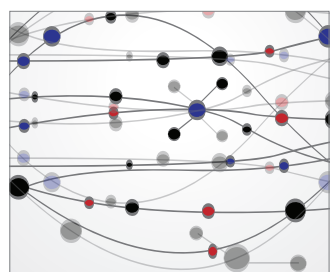

\section{The Scientific} World Journal
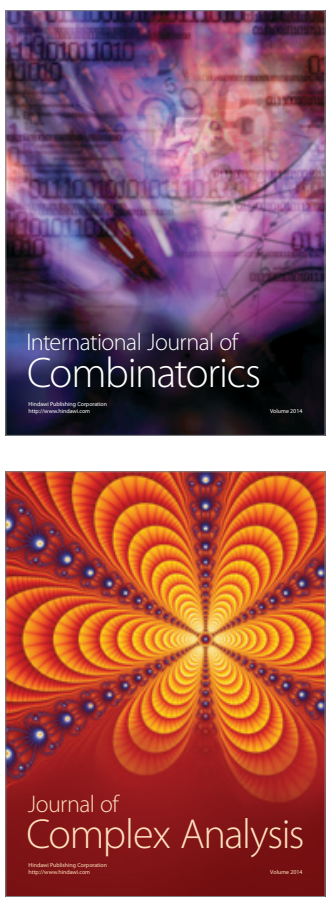

International Journal of

Mathematics and

Mathematical

Sciences
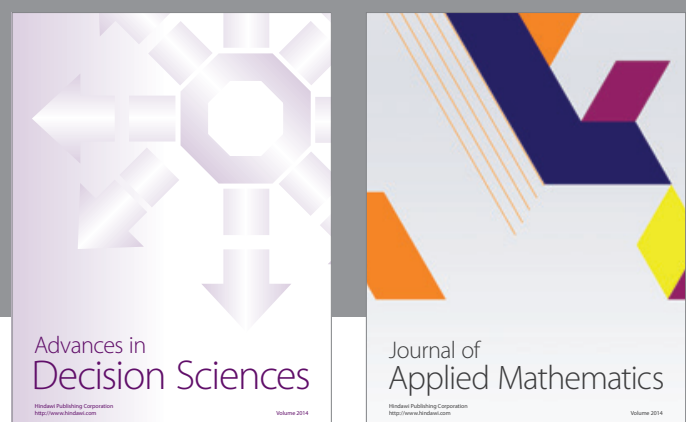

Journal of

Applied Mathematics
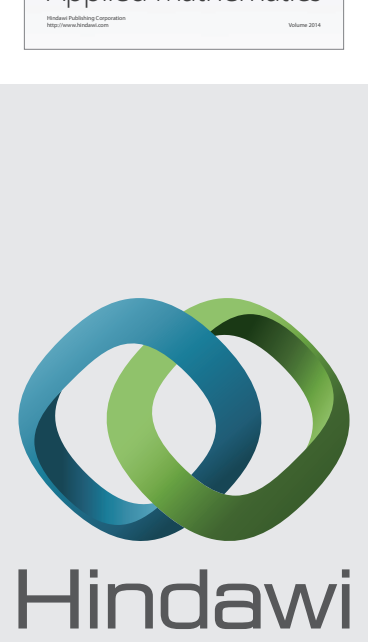

Submit your manuscripts at http://www.hindawi.com
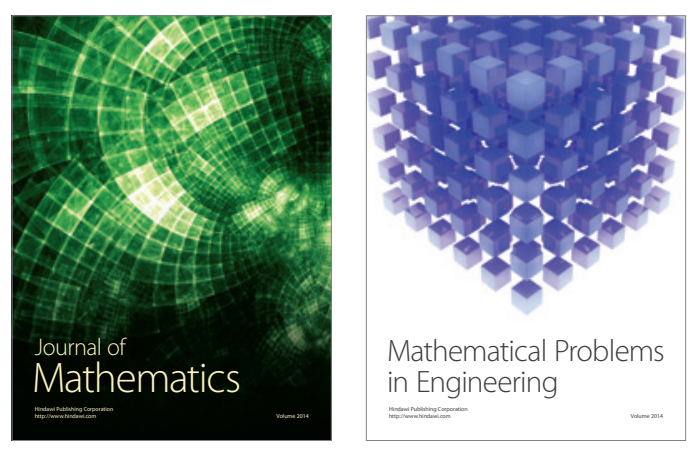

Mathematical Problems in Engineering
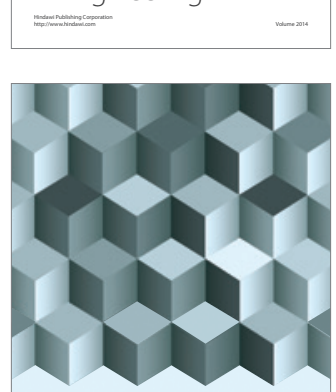

Journal of

Function Spaces
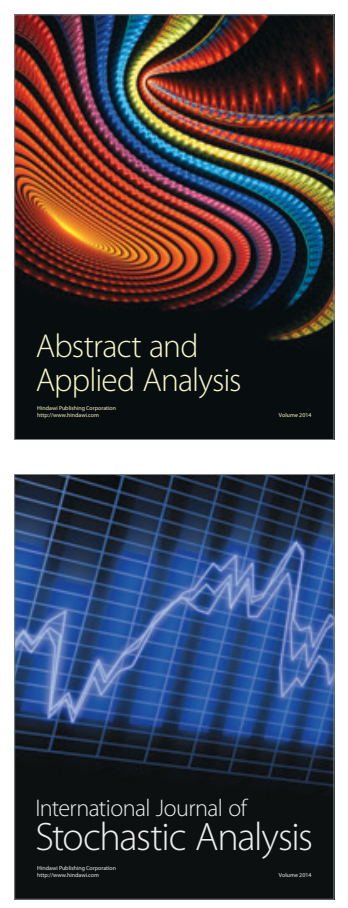

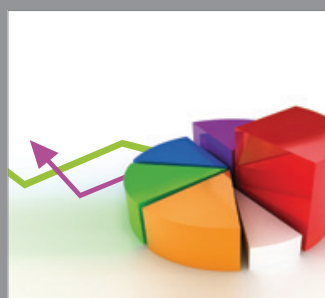

ournal of

Probability and Statistics

Promensencen
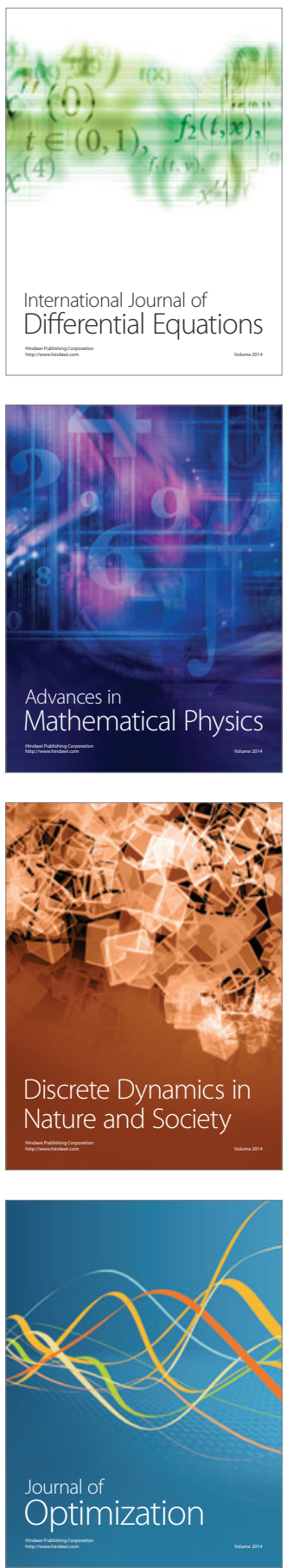